\title{
The effect of subsidies on healthful consumption: Systematic review and data synthesis
}

Abu-Hijleh $\mathrm{F}^{1}$ and Abu-Raddad LJ $\mathrm{LJ}^{2,3,4^{*}}$

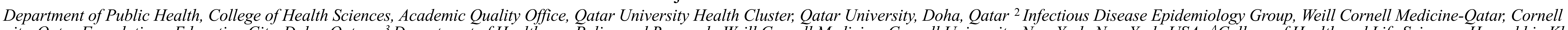

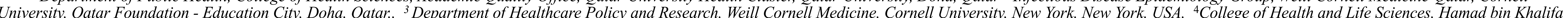

\section{Background}

- Prevalence of obesity and overweight have risen globally resulting in an unrestrained epidemic.

- Main contributing factor is thought to be a change in foodrelated consumer purchasing behavior.

- Health policy makers are seeking evidence-based approaches in an effort to alleviate the significant morbidity and mortality associated with obesity and overweight.

\section{Objective}

- This study focuses on reviewing and aggregating all available evidence on the subsidization of healthy foods as a fiscal tool to aid in the public health response to this epidemic.

\section{Methodology}

- A systematic search of the major international databases was conducted looking for studies on healthy food subsidies.

Relevant studies were synthesized into a table reviewing study design, outcomes and measures, in addition to conclusions (Table 1 and Fig. 1).

Key messages and overall trends were examined and presented, along with an assessment of the overall limitations and advantages of the available literature (Fig. 2).

\section{Results}

- Review yielded 25 reports, of which 12 studies were deemed to be relevant yielding a total of 63 measures.

- Price incentives for healthy foods combined with restrictions on unhealthy foods yielded the most promising results.

- A wide range of results ranging from $8 \%$ decrease to $136 \%$ increase reflected the heterogeneity in study design, type of intervention implemented, and outcomes reported.

Fig. 1: Results of outcome and measures.

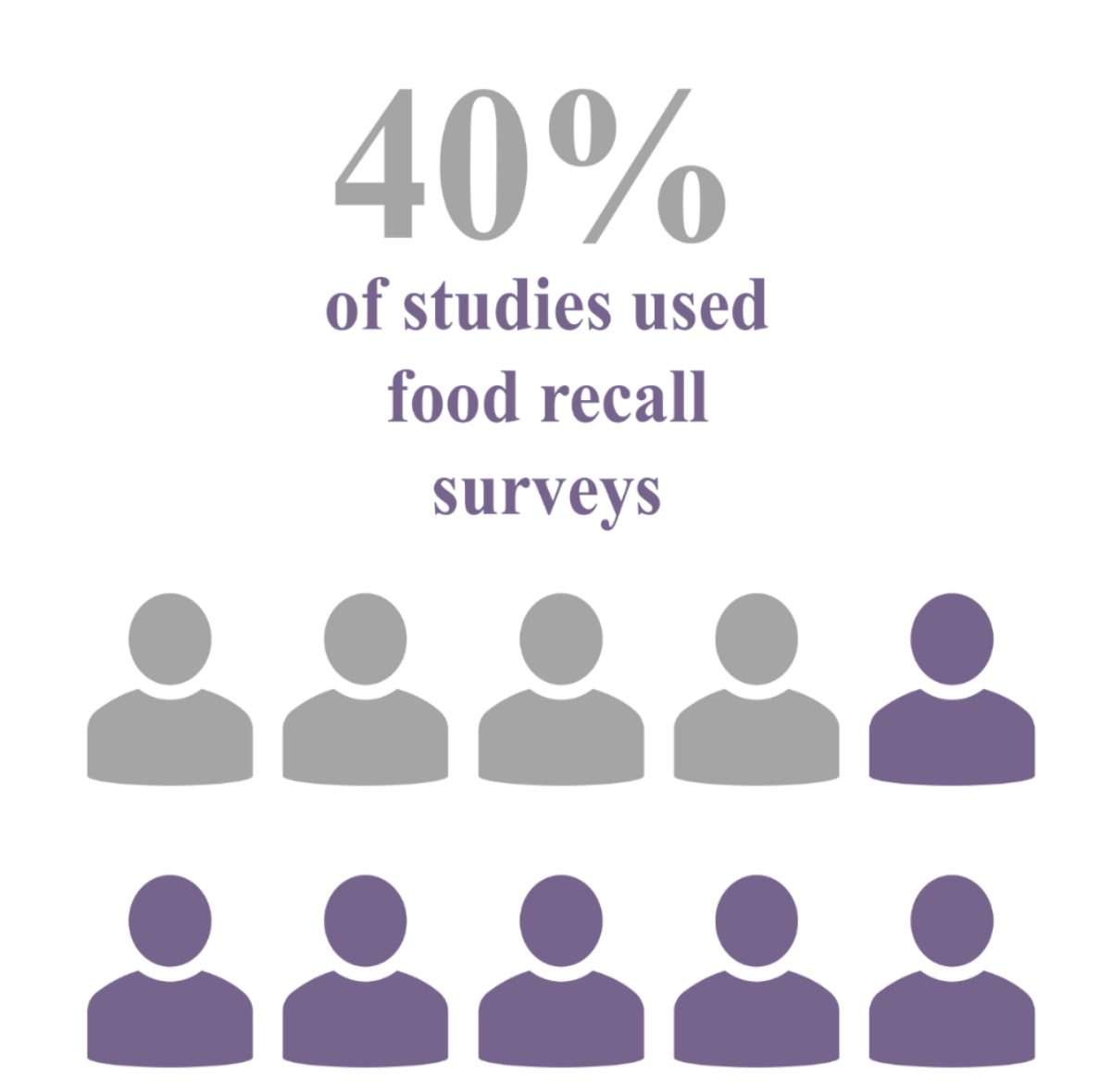

\section{Policy suggestions}

- Results suggest that multi-faceted "combination" interventions appear to be the most promising in terms of real changes to purchasing behavior.

- Surprisingly, education appeared to have little to no effect on outcomes.

- Important considerations include the significant heterogeneity in reported outcomes, the notable lack of studies assessing for an overall dietary and nutritional profile, and unknown cost effectiveness.

- In studies that assessed changes long term, effects were either absent or attenuated suggesting a sustainability challenge.

- Policy makers should consider these limitations and further expand the scope of fiscal interventions to include strategies to discourage the purchase of less healthy foods in addition to focusing on healthy foods.

Table 1: Study outcome measures on subsidies for healthy foods

\begin{tabular}{|c|c|c|c|c|c|c|c|}
\hline Study & \begin{tabular}{|l|l|} 
Interventio \\
In type
\end{tabular} & Amount & Outcome & Measurement & Effect & Conclusion & Notes \\
\hline $\begin{array}{l}\text { Andreyeva, } \\
2015\end{array}$ & Vouchers & $\begin{array}{l}\text { SUS } 6 \text { - \$US } 10 \\
\text { on fruit and veg }\end{array}$ & $\begin{array}{l}\text { Volume of fresh } \\
\text { fruit and vegetable } \\
\text { purchased }\end{array}$ & $\begin{array}{l}\text { Electronic } \\
\text { sales data }\end{array}$ & $\begin{array}{l}+\mathbf{1 7 . 5 \%} \text { fresh } \\
\text { vegetable purchase, } \\
+\mathbf{2 8 . 6 \%} \% \text { fresh fruit }\end{array}$ & $\begin{array}{l}\text { Efforts to encourage consumption } \\
\text { of } \\
\text { fruits and vegetables by people } \\
\text { receiving federal food assistance } \\
\text { are paying off }\end{array}$ & $\begin{array}{l}\text { - } 3 x \text { times as much use of } \\
\text { vouchers for fruit } \\
\text { - Assessed for substitution } \\
\text { effects } \\
\text { - Compared to baseline }\end{array}$ \\
\hline $\begin{array}{l}\text { Andreyeva, } \\
2016\end{array}$ & Vouchers & $\begin{array}{l}\text { \$US } 6 \text { - \$US } 10 \\
\text { on fruit and veg }\end{array}$ & $\begin{array}{l}\text { Volume of healthy } \\
\text { and non-healthy } \\
\text { food purchased }\end{array}$ & $\begin{array}{l}\text { Electronic } \\
\text { sales data }\end{array}$ & $\begin{array}{l}\mathbf{+ 3 . 9 \%} \text { increase } \\
\text { healthy foods, } \mathbf{- 1 . 8 \%} \\
\text { decrease in moderation } \\
\text { foods, -24.7\% } \\
\text { decrease moderation } \\
\text { beverages }\end{array}$ & $\begin{array}{l}\text { Efforts to encourage healthy } \\
\text { eating by people receiving federal } \\
\text { food assistance are paying off }\end{array}$ & $\begin{array}{l}\text { - Assessed for substitution } \\
\text { effects } \\
\text { - Compared to baseline }\end{array}$ \\
\hline Ball, 2015 & $\begin{array}{l}\text { Price } \\
\text { reduction } \\
\text { and skill } \\
\text { building }\end{array}$ & $\begin{array}{l}20 \% \text { discount } \\
\text { on fruit and veg }\end{array}$ & $\begin{array}{l}\text { Volume of fruit and } \\
\text { vegetable purchase }\end{array}$ & $\begin{array}{l}\text { Electronic } \\
\text { sales data }\end{array}$ & $\begin{array}{l}+\mathbf{1 5 \%} \% \text { increase } \\
\text { vegetable purchases, }+ \\
\mathbf{3 5 \%} \text { increase fruit } \\
\text { purchases }\end{array}$ & $\begin{array}{l}\text { Findings show that price } \\
\text { modifications can directly } \\
\text { increase produce purchases }\end{array}$ & $\begin{array}{l}\text { - Multi-arm and control group } \\
\text { - Sugar and diet beverages } \\
\text { increased } \\
\text { - Increases not maintained } 6 \\
\text { month post intervention }\end{array}$ \\
\hline $\begin{array}{l}\text { Brimblecombe, } \\
2017\end{array}$ & $\begin{array}{l}\text { Price } \\
\text { reduction } \\
\text { and } \\
\text { education }\end{array}$ & $\begin{array}{l}20 \% \text { discount } \\
\text { on fruit, veg, } \\
\text { diet drinks }\end{array}$ & $\begin{array}{l}\text { Volume of fruit and } \\
\text { vegetable purchase }\end{array}$ & $\begin{array}{l}\text { Weekly sales } \\
\text { data }\end{array}$ & $\begin{array}{l}+\mathbf{1 2 . 7 \%} \text { increase in } \\
\text { fruit and vegetable } \\
\text { purchased }\end{array}$ & $\begin{array}{l}\text { A } 20 \% \text { discount can only increase } \\
\text { fruit and vegetable purchases to } \\
\text { help protect against obesity and } \\
\text { diet } \\
\text { related disease to a certain extent }\end{array}$ & $\begin{array}{l}\text { - Larger effect on fruit yet } \\
\text { modest } \\
\text { - Education has small additive } \\
\text { effect }\end{array}$ \\
\hline Cardenas, 2015 & $\begin{array}{l}\text { Price } \\
\text { reduction }\end{array}$ & $\begin{array}{l}33 \% \text { discount } \\
\text { on fruit }\end{array}$ & $\begin{array}{l}\text { Number of fruits } \\
\text { consumed }\end{array}$ & $\begin{array}{l}\text { Food recall } \\
\text { surveys }\end{array}$ & $\begin{array}{l}\mathbf{+ 1 3 6 \%} \text { increase fruits } \\
\text { sold }\end{array}$ & $\begin{array}{l}\text { Pricing is a barrier to fruit } \\
\text { purchasing, discounts lead to } \\
\text { significant increases }\end{array}$ & $\begin{array}{l}\text { Very small sample size, } \\
\text { short duration }\end{array}$ \\
\hline Ferguson, 2017 & $\begin{array}{l}\text { Price } \\
\text { reduction }\end{array}$ & $\begin{array}{l}10 \% \text { discount } \\
\text { on fruit, veg, } \\
\text { diet drinks }\end{array}$ & $\begin{array}{l}\text { Volume of fruit and } \\
\text { vegetable purchase }\end{array}$ & $\begin{array}{l}\text { Electronic } \\
\text { sales data }\end{array}$ & $\begin{array}{l}-\mathbf{- 3 \%} \text { decrease in fruits } \\
\text { purchased and } \mathbf{- 8 \%} \\
\text { decrease in vegetables } \\
\text { purchased }\end{array}$ & $\begin{array}{l}\text { no discernible effect was evident, } \\
\text { due to inadequate design and } \\
\text { communication of discount } \\
\text { promotion, and probably } \\
\text { inadequate magnitude of discount }\end{array}$ & $\begin{array}{l}\text { - Small sample and limited } \\
\text { discount } \\
\text { - Non significant p value and } \\
\text { very wide CIs }\end{array}$ \\
\hline Gorham, 2015 & $\begin{array}{l}\text { Farmers } \\
\text { markets and } \\
\text { price } \\
\text { reduction }\end{array}$ & $\begin{array}{l}15 \% \text { to } 25 \% \text { on } \\
\text { fruit and veg }\end{array}$ & $\begin{array}{l}\begin{array}{l}\text { Fruit and vegetable } \\
\text { intake }\end{array} \\
\text {. }\end{array}$ & $\begin{array}{l}\text { Food recall } \\
\text { surveys }\end{array}$ & $\begin{array}{l}\mathbf{+ 0 . 4 8} \text { cups/d increase } \\
\text { fruit and vegetable } \\
\text { intake }\end{array}$ & $\begin{array}{l}\text { Intervention effective at } \\
\text { increasing consumption of fruits } \\
\text { and vegetables among racially } \\
\text { and ethnically diverse low- } \\
\text { income children }\end{array}$ & - No baseline to compare to \\
\hline Harnack, 2016 & $\begin{array}{l}\text { Price } \\
\text { reduction } \\
\text { with } \\
\text { restriction }\end{array}$ & $\begin{array}{l}30 \% \text { discount } \\
\text { on fruit and } \\
\text { vegetable }\end{array}$ & $\begin{array}{l}\text { Energy intake and } \\
\text { fruit consumption }\end{array}$ & $\begin{array}{l}\text { Food recall } \\
\text { surveys }\end{array}$ & $\begin{array}{l}-96 \mathbf{k c a l} / \mathbf{d} \text {, and }+0.2 \\
\text { servings fruit/day }\end{array}$ & $\begin{array}{l}\text { A number of favorable changes } \\
\text { were observed in the incentive } \\
\text { plus restriction condition } \\
\text { suggesting an improvement in } \\
\text { nutritional quality }\end{array}$ & $\begin{array}{l}\text { - Multi arm and control group } \\
\text { Combination price and } \\
\text { restriction group most } \\
\text { siggificant changes }\end{array}$ \\
\hline Kristal, 1997 & $\begin{array}{l}\text { Coupons } \\
\text { and } \\
\text { promotion }\end{array}$ & $\begin{array}{l}50 \text { cent off fruit } \\
\text { and vegetable }\end{array}$ & $\begin{array}{l}\text { Fruit and vegetable } \\
\text { consumption }\end{array}$ & $\begin{array}{l}\text { Food recall } \\
\text { surveys }\end{array}$ & $\begin{array}{l}\mathbf{+ 8 . 8 \%} \text { increase in fruit } \\
\text { consumption, }+\mathbf{1 1 . 1} \% \\
\text { increase in vegetable } \\
\text { consumption }\end{array}$ & $\begin{array}{l}\text { More powerful interventions are } \\
\text { probably necessary to induce } \\
\text { shoppers to purchase and } \\
\text { consume more fruits and } \\
\text { vegetables. }\end{array}$ & $\begin{array}{l}\text { - Results compared to } \\
\text { baseline, compared to } \\
\text { control group there is no } \\
\text { impact } \\
\text { - Not a strong financial } \\
\text { incentive }\end{array}$ \\
\hline Mooney, 2014 & $\begin{array}{l}\text { Price } \\
\text { reduction } \\
\text { (vending } \\
\text { machine) }\end{array}$ & $\begin{array}{l}25 \% \text { discount } \\
\text { healthy vending } \\
\text { machine snacks }\end{array}$ & $\begin{array}{l}\text { Healthy snack } \\
\text { purchase }\end{array}$ & Sales receipts & $\begin{array}{l}+\mathbf{3 4 . 5 \%} \text { increase site } \\
\mathrm{A},+\mathbf{+ 1 . 8 \%} \text { increase site } \\
\mathrm{B}, \mathbf{+ 1 2 . 9 \%} \text { increase } \\
\text { site } \mathrm{C},+\mathbf{1 9 . 6 \%} \\
\text { increase site } \mathrm{D} \text { for } \\
\text { healthy snack purchase }\end{array}$ & $\begin{array}{l}\text { Price-discounting can be an } \\
\text { effective means of initiating } \\
\text { more nutrititiously balanced } \\
\text { vending choices among diverse } \\
\text { groups of } \\
\text { employees in a Scottish } \\
\text { population }\end{array}$ & $\begin{array}{l}\text { - Changes not followed long } \\
\text { term, unsure about } \\
\text { sustainability } \\
\text { - Site B had very high } \\
\text { baseline uptake }(71 \%)\end{array}$ \\
\hline $\begin{array}{l}\text { Ni Mhurchu, } \\
2010\end{array}$ & $\begin{array}{l}\text { Price } \\
\text { reduction } \\
\text { with } \\
\text { education }\end{array}$ & $\begin{array}{l}12.5 \% \text { discount } \\
\text { on healthy } \\
\text { foods }\end{array}$ & $\begin{array}{l}\text { Fruit and vegetable } \\
\text { purchase, healthy } \\
\text { food purchase }\end{array}$ & $\begin{array}{l}\text { Electronic } \\
\text { sales data }\end{array}$ & $\begin{array}{l}\mathbf{1 1 0 \%} \text { increase fruit } \\
\text { and vegetable } \\
\text { purchased, }+\mathbf{1 1} \% \\
\text { increase overall } \\
\text { healthy food purchased }\end{array}$ & $\begin{array}{l}\text { Significant and sustained effect } \\
\text { of discounts on food purchases } \\
\text { suggests that pricing strategies } \\
\text { hold promise as a means to } \\
\text { improve population diets. }\end{array}$ & $\begin{array}{l}\text { - Multi arm and control group } \\
\text { - Education has no effect } \\
\text { Changes sustained after six } \\
\text { months but attenuated }\end{array}$ \\
\hline Olsho, 2016 & $\begin{array}{l}\text { Price } \\
\text { reduction }\end{array}$ & $\begin{array}{l}30 \% \text { discount } \\
\text { on fruit and } \\
\text { vegetables }\end{array}$ & $\begin{array}{l}\text { Fruit and vegetable } \\
\text { consumption }\end{array}$ & $\begin{array}{l}\text { Food recall } \\
\text { surveys }\end{array}$ & $\begin{array}{l}+\mathbf{2 3} \% \text { increase in fruit } \\
\text { consunpution, ++30\% } \\
\text { increase in vegetable }\end{array}$ & $\begin{array}{l}\text { Health incentives significantly } \\
\text { increased fruit and vegetable } \\
\text { intake among participants, }\end{array}$ & \\
\hline
\end{tabular}

Fig. 2: Limitations and advantages of the available literature.

\section{Limitations and sources of bias}

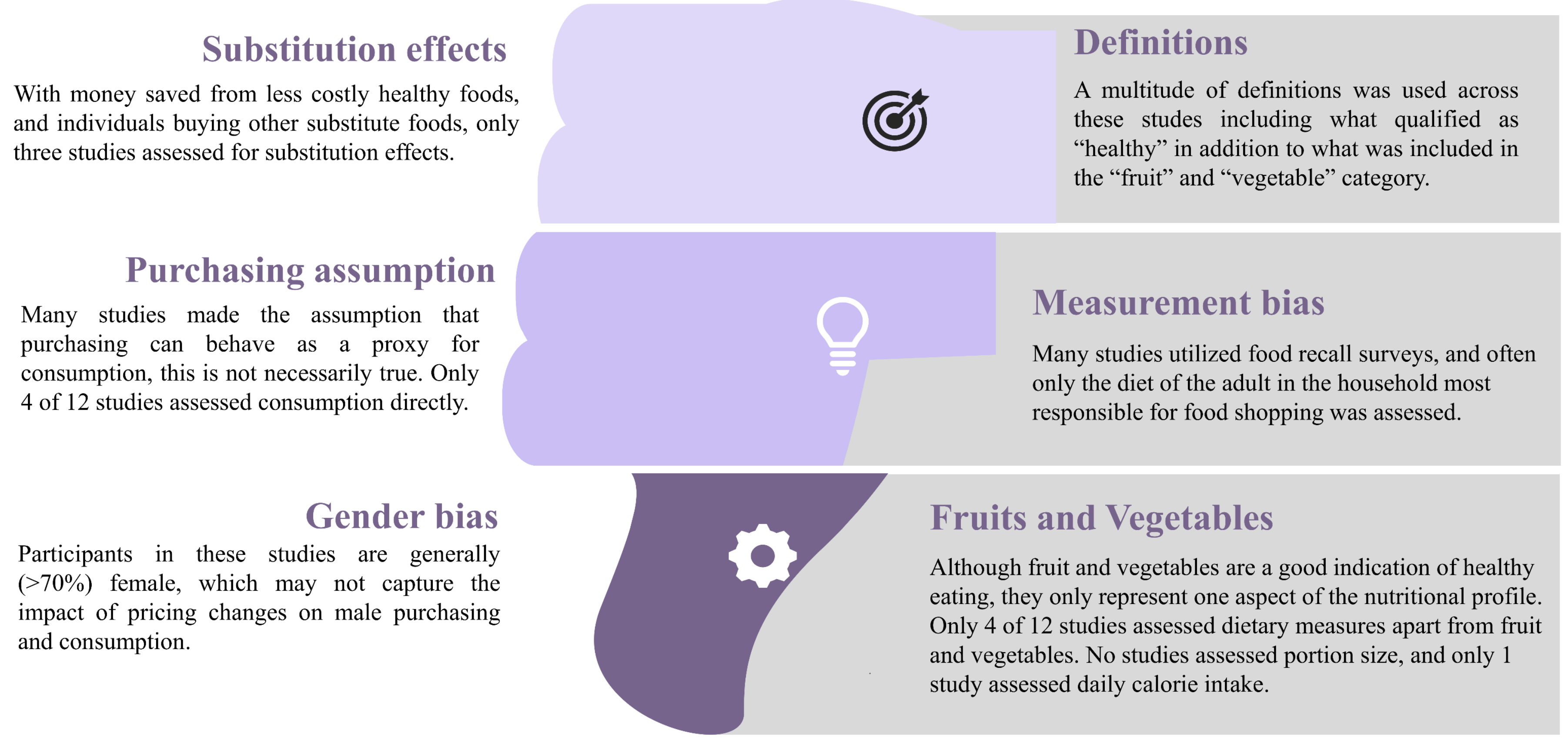

\section{Advantages}

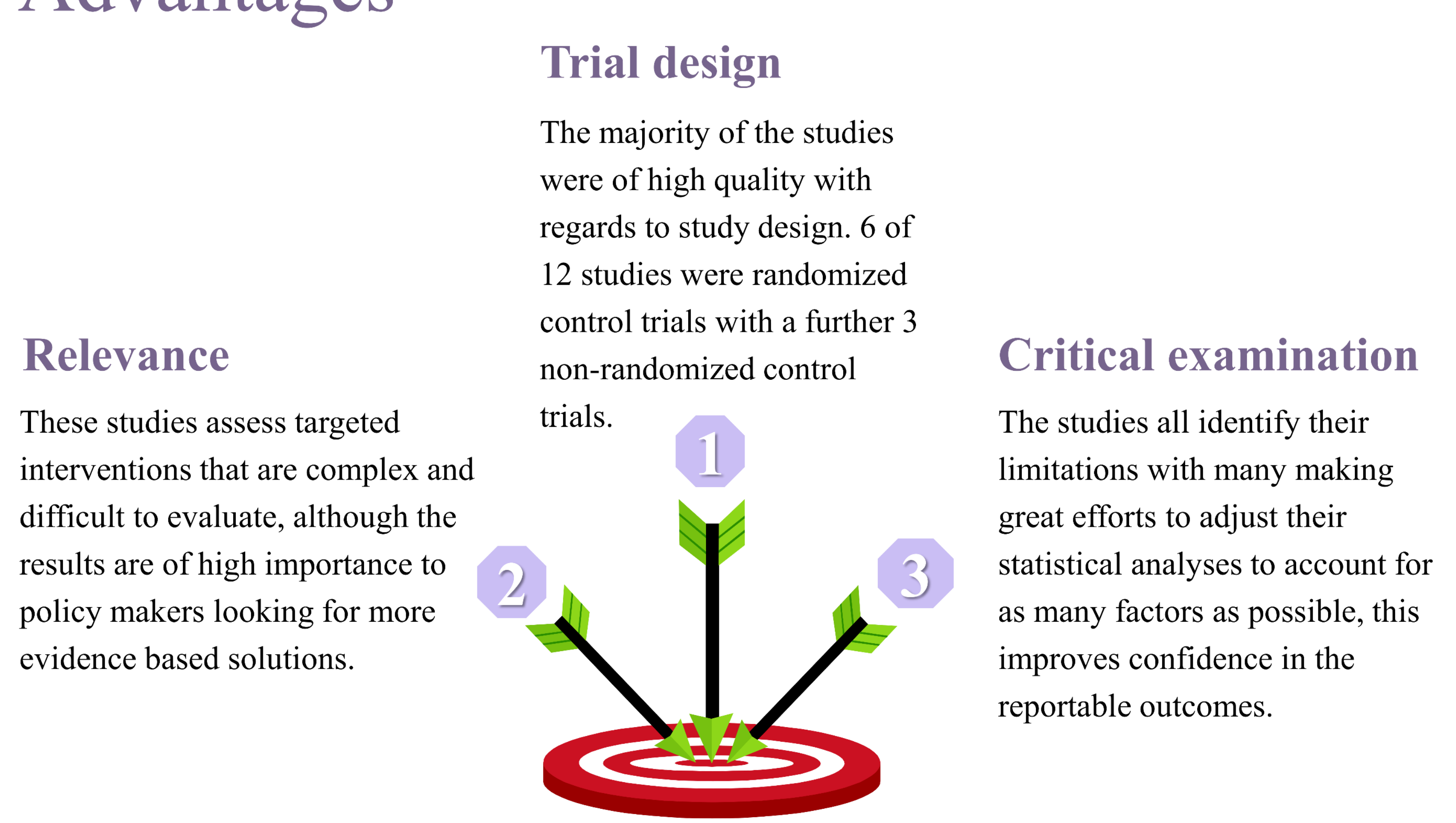

